\title{
Wind-Aided Flame Spread Under Oblique Forced Flow
}

\author{
J. M. TIZÓN, J. J. SALVÁ, and A. LIÑÁN* \\ Universidad Politécnica de Madrid, E. T. S. I. Aeronáuticos, Madrid, Spain
}

\begin{abstract}
The wind-aided flame spread process along a solid fuel rod under oblique forced flow is analyzed in absence of gravity or when the forced flow dominates the gravity-induced flow. The transverse velocity is large enough to ensure that mixing of the fuel vapors and air occurs in a thin boundary layer surrounding the fuel rod and we can use the boundary layer approximation to describe the gas-phase chemical reaction and downwind flame spread process. A global, second-order, Arrhenius expression is employed to describe the gas-phase reaction, while the solid surface gasification reaction is modeled in terms of a constant pyrolysis temperature. The solid is heated by the hot gases convected from the flame by the axial component of the velocity in the direction of the flame spread. The solid will be considered thermally thick, assuming the thickness of the heated layer in the solid to be small compared with the rod radius. The analysis determines the flame spread velocity and the flow structure in the flame front region. The analysis also shows that flame spread is not possible at large flow velocities due to finite rate effects, while at low velocities the gas-phase reaction is diffusion-controlled. By including radiation losses from the surface a flame spread limit, at low velocities, is also found in the present analysis.
\end{abstract}

\section{NOMENCLATURE}

\section{Latin symbols}

$a$

A

$B_{g}$

$c_{p}$

$c_{s}$

$D$

$\bar{D}$

$E_{g}$

$k$

Le

$L_{v}$

$\dot{m}_{s}$

$n$

$N$

$\operatorname{Pr}$

$q$

$q_{F}$

$q_{g, s}$

$\dot{r}$

$\operatorname{Re}$

rod radius

strain rate reaction

pressure

reaction

ratio fuel preexponential factor for gas-phase

gas-phase specific heat at constant

solid-phase specific heat

Damköhler number

modified Damköhler number

activation energy for gas-phase

thermal conductivity

Lewis number

heat of pyrolysis

solid-phase surface mass flux

exponent in $\mathrm{T}$ dependence of $\mathrm{k}$

gas to solid characteristic thickness

Prandtl number

nondimensional heat of reaction, $q=q_{F} / c_{p} T_{\infty}$

heat of reaction per unit of mass

nondimensional heats

solid surface recession rate

Reynolds number, $R e=a v_{\infty} / \nu_{\infty}$

*Corresponding author: E-mail: tizon@prop.dmt.upm.es

$R e_{c} \quad$ characteristic transversal Reynolds number, $R e_{c}=A_{e} a^{2} / \alpha_{\infty}$

$S$

$S$

$S_{R} \quad$ radiation parameter

$\bar{S}_{R}$

$T$

$T_{a}$

$U_{L}$

$u, v, w$

$V, W$

$\dot{w}_{F}$

$W_{f}$

$Y$

$x, y, z \quad$ boundary layer coordinates

$\ell$ characteristic length of the front

\section{Greek Symbols}

$\alpha$

$\beta_{1,2}$

$\chi$

$\delta$

$\epsilon$

$\kappa$ thermal diffusion coefficient coupling functions gas to solid ratio of specific heats at constant pressure, $\chi=c_{p} / c_{s}$ characteristic thickness emissivity factor strain rate constant coefficient 


\section{Subscripts}

$\begin{array}{ll}\infty & \text { infinite } \\ c & \text { characteristic value } \\ e & \text { external } \\ f & \text { flame } \\ F & \text { fuel } \\ g & \text { gas phase } \\ O & \text { oxygen } \\ v & \text { pyrolysis } \\ s & \text { surface of solid }\end{array}$

\section{Superscripts and Tildes}

Solution at $z \rightarrow+\infty$ (frozen)
Solution at $z \rightarrow-\infty$ (equilibrium)

\section{INTRODUCTION}

The subject of flame spread over condensed fuels burning in air has received considerable attention in the literature due to its implications for fire safety. Early reviews of the subject were given by Williams [1] and [2], Fernandez-Pello and Hirano [3]. More recent reviews are given by Wichman [4], di Blasi [5], and Sigirano and Schiller [6].

The effects on flame spread of the convective transport of heat and reacting species, due to a forced flow or to the flow induced by gravity, have been studied using approximate or numerical simulations of the complete equations. Most of these studies, after the pioneering work of de Ris [7] who considered the chemical reaction to be diffusion-controlled, have dealt with the special cases for which the flow, or gravity, is parallel to the solid surface. In these cases the flame spread velocity is constant,

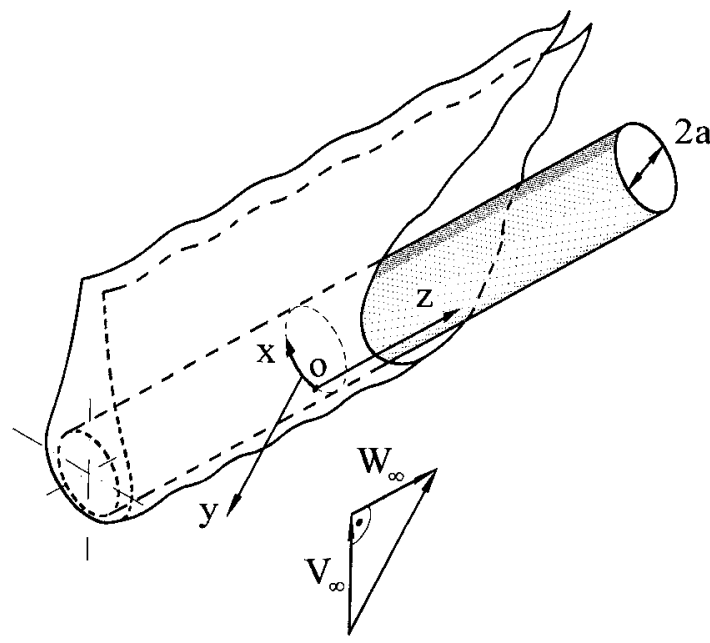

Fig. 1. Schematic representation of the flame spread process along a fuel rod with forced flow velocity oblique to the rod. The reference frame is moving relative to the rod with the flame spread velocity $W_{j}$.

whereas in the cases of flame propagation upwards (see for example Williams [8]) or downwind propagation when the flow is parallel to the solid surface (see for example [9] and [10]), the flame spread processes are self-accelerating.

In this work we shall analyze the threedimensional wind-aided flame spread process along a fuel rod, of radius $a$, in the general case, not considered previously in the literature, when the forced flow is oblique to the rod (Fig. 1). In this case if by external heating, in a certain region of the fuel rod, we ignite the diffusion flame between the vaporizing fuel and air, then the flame will spread along the rod by means of two fronts: one against the longitudinal component of the external velocity, and the other downwind, aided by the flow.

The flame front propagation against the flow is dominated by the structure of the flame front region, where, as shown in the analysis of de Ris [7], a balance is found between convection and upstream heat conduction. Therefore, the analysis of this upwind flame front region, which we do not analyze in this paper, can not be carried out using the boundary layer approximation. We shall neither analyze the case of pure transverse flow where the two flame fronts have the same structure, and can not be described using the boundary layer approximation.

However, in the case of flame propagating 
downwind, which we analyze in this paper, accounting for the effects of the crosswise component of the velocity, we find that, due to this component, the heat transported by the axial component of the velocity is partially balanced by the heat transported by the crosswise component limiting the heating region of the front to a zone of the order of the radius of the rod. Then, there is a constant flame spread velocity, $W_{f}$, to be determined by our analysis. If we use a reference frame moving relative to the rod with the velocity $W_{f}$, which is very small compared with the axial component $w_{\infty}$ of the external velocity, the flame front structure becomes quasi-steady. Then the quasi-steady form of the equations can be used, both for the solid and the gas phases. Along the rod, we can distinguish three different regions: a flame front region, of characteristic size $a$, separates a nonburning region downstream (on the right hand side of Fig. 1) where the flow is two-dimensional, from an upstream burning region (left side of Fig. 1) where the flow structure is also two-dimensional if the regression velocity of the surface is small compared with the flame spread velocity.

The structure of the upstream burning region, in the stagnation point zone, is dependent only on the transverse velocity component $v_{\infty}$, and it has been often analyzed for the case where the Reynolds number based on the rod radius and $v_{\infty}$ is large compared with unity; then, mixing and chemical reaction of the fuel with the air take place in a thin boundary layer [11]. With this large Reynolds number assumption, which we shall use in our work, viscosity, heat conduction, and diffusion effects are also confined in the flame front region to a thin boundary layer. The gas flow outside this layer is inviscid, with the constant temperature (and density) of the air stream, and only influenced by the effects of combustion in the boundary layer through their effect on the boundary layer separation and subsequent formation of the von Karman street in the wake of the rod. Nevertheless, for reasons that will become more clear from the analysis, we shall neglect the effects of the changes in wake structure on the flame spread velocity. Then, as in the flow past swept wings, outside the boundary layer the axial flow velocity is constant equal to $w_{\infty}$ and the transverse component of the velocity at the edge of the boundary layer is proportional to $v_{\infty}$ independent of $w_{\infty}$. Within the boundary layer the flow structure is determinated by its behavior in the leading stagnation line region, because upwind information is eliminated with the boundary layer approximation. Thus, the flame spread velocity will be determinated by the boundary layer flow structure in the zone around the stagnation line, which will be analyzed below.

The importance of the kinetic effects on flame spread was first taken into account by Frey and T'ien [12], in a numerical study with an Arrhenius reaction of the second order for the gas phase and a first order for the solid pyrolysis. The computations show that the flame spread process is extinguished at low Damköhler numbers. Frey and T'ien [12] were the first to correlate the flame spread velocity with the Damköhler number.

The kinetics of the gas-phase reaction will also be modeled in our analysis by an exothermic one-step Arrhenius reaction between the pyrolyzed fuel and the oxygen of the air. The solid pyrolysis process will be modeled in terms of a pyrolysis temperature $T_{v}$; so that no pyrolysis is considered to occur at solid surface temperatures below $T_{v}$, and the fuel temperature will remain constant, equal to $T_{v}$, if pyrolysis occurs. When the solid surface temperature reaches the value $T_{v}$ the solid begins to gasify at a rate controlled by the heat transfer process. This simplified model describes well the pyrolysis process when the gasification reaction is endothermic with a large activation energy. We shall consider that the regression velocity of the interface is small compared with $W_{f}$, and thus the relative changes in the rod radius are small in the flame front region.

The temperature field in the heated region of the solid is steady when seen with the reference frame moving with the front. In this frame the solid moves toward the burning region with a velocity $W_{f}$. The heat reaching the solid from the gas phase penetrates the solid by heat conduction reaching a layer with a thickness that, in the flame front region, we consider to be small compared with the rod radius, $a$; so that the solid behaves as thermally thick.

We shall leave out of the analysis the buoyancy force due to gravity; this is justified, for example, for flame spread in a microgravity environment, or more precisely when the gravityinduced velocity, of order $\sqrt{g a}$, is small compared 
with $v_{\infty}$. We do not include in the analysis the effects of radiation from the gas phase in the flame spread process; the heating due to convection will dominate the process when the flow velocity is large enough. But we shall take into account the effect of radiation losses from the solid surface to describe the possible extinction of the flame spread process when the forced flow is reduced leading to a reduction of the heat coming from the gas phase to vaporize the fuel; however our analysis will only be applicable if the extinction Reynolds number is still large compared with unity. These extinction processes are similar to those anticipated theoretically by T'ien [13] for the diffusion flame in the stagnation point region adjacent to a solid fuel and the case described experimentally by Olson [14], in the case of propagation opposite to the flow in a microgravity environment.

Based on order of magnitude estimates for this model, some approximations are made to simplify the numerical solution of the resulting problem. A numerical integration is performed by means of an implicit finite difference method based on the box method of Keller [15, 16] and a special mesh adaptation procedure is used to improve the description of very thin flames at high Damköhler numbers.

Results are presented, first, for the case where the gas-phase reaction is infinitely fast, because it shares the main characteristics of the spread process and it is the appropriate starting point to evaluate finite chemical kinetic effects. Comparison is then made with the cases where finite rate chemistry is included, and an extinction regime is identified at low Damköhler numbers. Finally, some results are also presented for the effects of radiation losses from the surface. We show how the flame spread velocity is reduced, at high Damköhler numbers, due to radiation losses, with decreasing values of the transverse velocity. The flame spread velocity is positive only for values of the transverse velocity above a critical value.

\section{FORMULATION}

For the description of the reacting boundary layer we shall use coordinates with the origin placed at the point on the stagnation line, of the external transverse inviscid flow, where the sur- face temperature first reaches the gasification value $T_{v}$. The $x$ coordinate is the distance from the stagnation line along the rod surface and transverse to the rod axis, the $y$ coordinate is normal to the solid surface, and the $z$ coordinate is parallel to the rod axis, positive in the direction of flame spread. In terms of these coordinates the surface temperature at $x=0$, remains equal to $T_{v}$, for $z \leq 0$, and it is unknown, $T_{s}(z)$ smaller than $T_{v}$, for $z>0$.

Far from the cylinder, the oblique flow is assumed to have a transverse component $v_{\infty}$ and a longitudinal component $w_{\infty}$ of the same order of magnitude; with the transverse Reynolds number, $a v_{\infty} / \nu_{\infty}$, large compared with unity. For these conditions, the flow outside a thin boundary layer, which may separate on the lee side of the rod, will be irrotational and will determine the external boundary conditions for the equations describing the flow in the laminar boundary layer, of thickness $a / \sqrt{R e}$. Because for $x / a$ $\ll 1, u_{e}=A_{e} x$, where $A_{e}$, of order $v_{\infty} / a$, is the crosswise inviscid flow strain rate at the stagnation point, these equations have, for $x / a \ll 1$, a similarity solution, with the three components of the velocity $u, v$, and $w$ in the form,

$u / x \approx A(y, z), v \approx V(y, z), w \approx W(y, z)$,

where the strain rate $A$ and the velocities $V$ and $W$, are only functions of $y$ and $z$; the same is true for the rest of the flow variables,

$$
\begin{aligned}
Y_{F} & \approx Y_{F}(y, z), Y_{O} \\
& \approx Y_{O}(y, z), T \\
& \approx T(y, z) \text { and } \rho \\
& \approx \rho(y, z) .
\end{aligned}
$$

The similarity solution corresponds to the first term of an expansion of the flow variables in powers of $(x / a)^{2}$.

Due to the parabolic character of the boundary layer equations, the structure of the flow at small values of $x / a$ is independent of the downstream evolution of the boundary layer, aside from the effects of the boundary layer separation, which we will neglect. The analysis of the flow in the upstream similarity region of the boundary layer will determine the flame spread 
velocity; the flow downstream is slave to the flow in the stagnation region.

Using the previous considerations, the gasphase reacting boundary layer equations, valid for $y>0$, take the form:

$$
\begin{gathered}
\rho A+\frac{\partial(\rho V)}{\partial y}+\frac{\partial(\rho W)}{\partial z}=0 \\
\rho A^{2}+\rho V \frac{\partial A}{\partial y}+\rho W \frac{\partial A}{\partial z}=\rho_{\infty} A_{e}^{2} \\
+\operatorname{Pr} \frac{\partial}{\partial y}\left(\frac{k_{g}}{c_{p}} \frac{\partial A}{\partial y}\right)
\end{gathered}
$$

$\rho V \frac{\partial Y_{O}}{\partial y}+\rho W \frac{\partial Y_{O}}{\partial z}=\frac{\partial}{\partial y}\left(\frac{k_{g}}{c_{p} L e_{O}} \frac{\partial Y_{O}}{\partial y}\right)-s \dot{w}_{F}$

$\rho V \frac{\partial T}{\partial y}+\rho W \frac{\partial T}{\partial z}=\frac{\partial}{\partial y}\left(\frac{k_{g}}{c_{p}} \frac{\partial T}{\partial y}\right)+\frac{q_{F}}{c_{p}} \dot{w}_{F}$

where $\operatorname{Pr}$ is the Prandtl number, $L e_{F}$ and $L e_{O}$ are the fuel and oxidizer Lewis numbers respectively, $k_{g}=k_{\infty}\left(T / T_{\infty}\right)^{n}$ is the gas-phase conductivity as a function of the temperature, $c_{p}$ is the specific heat, considered constant, at constant pressure, $s$ is the mass of oxygen consumed per unit mass of fuel in the single-step irreversible reaction $F+s O \rightarrow P$, and $q_{F}$ is the heat release per unit mass of fuel due to the reaction.

For the fuel consumption rate per unit volume and time $\dot{w}_{F}$, we use an Arrhenius expression

$\dot{w}_{F}=-\rho B_{g} Y_{F} Y_{O} e^{-E_{g} / R T}$,

where $B_{g}$ is the preexponential factor and $E_{g}$ is the activation energy of the gas-phase reaction.

The density $\rho$ is determined in terms of $T$ by the gas-phase equation of state that takes the simplified form

$\rho T=\rho_{\infty} T_{\infty}$,

because the spatial pressure variations can be neglected compared with the pressure itself, and we also consider the mean molecular mass to be constant and equal to that of the free stream air.

For the description of the temperature within the cylinder, we consider the case when the conductivity of the solid is small enough as to ensure that, in the flame spread region $(z \approx a)$, the thickness of the thermally heated layer of the solid is small compared with the rod radius $a$. In this case, the temperature distribution in the solid $(y<0)$ is given by

$\frac{\dot{m}_{s}(z)}{\rho_{s}} \frac{\partial T}{\partial y}-W_{f} \frac{\partial T}{\partial z}=\alpha_{s} \frac{\partial^{2} T}{\partial y^{2}}$,

where $\dot{m}_{s}(z)$ is the solid pyrolysis rate per unit surface and time, which will be zero for $z>0$; $\rho_{s}$ is the solid density, $W_{f}$ is the flame spread velocity, and $\alpha_{s}$ is the solid thermal diffusivity. The ratio $\dot{m}_{s} / \rho_{s}$ is the regression velocity $\dot{r}$ of the solid surface, which we consider to be small compared with $W_{f}$.

\section{Boundary Conditions}

The boundary conditions for this system of equations are the following: At the edge of the boundary layer the fluid variables tend to the values of the inviscid flow,

$A \rightarrow A_{e}, W \rightarrow W_{\infty}, Y_{O} \rightarrow Y_{O \infty}, Y_{F} \rightarrow 0$,

$T \rightarrow T_{\infty}$ at $y \rightarrow \infty$.

The analysis of the inviscid external flow gives $A_{e}=\kappa v_{\infty} / a$, where $\kappa$ is a constant coefficient, For a circular rod, $\kappa=2$ if we assume that there is no separation of the external irrotational inviscid flow; this value is slightly reduced due to the boundary layer separation. In the second condition of (12) we have replaced $\left(W_{f}+W_{\infty}\right)$ by $W_{\infty}$, anticipating that $W_{f} \ll W_{\infty}$.

For $y \rightarrow-\infty$, in the solid, outside the thermally heated layer, the temperature $T \rightarrow T_{\infty}$.

The boundary conditions at the interface correspond to the requirement that the solid and gas temperatures must be equal there. So that

$T_{0^{+}}=T_{0^{-}}=T_{s}(z)$ at $y=0$,

the surface temperature must be constant, $T_{s}(z)=T_{v}$, for $z<0$, while $T_{s}(z)<T_{v}$ must be obtained as part of the solution for $z>0$. 
The no slip condition for $A$ and $W$ at the interface leads to

$A=W=0$ at $y=0$,

where the surface condition for the longitudinal velocity has been simplified from $W=-W_{f}$, anticipating that $W_{f} \ll W_{\infty}$. Finally, the conservation of mass, species, and energy at the surface gives

$\{\rho V\}_{0^{+}}=\dot{m}_{s}(z)$,

$\left\{\dot{m}_{s}(z) Y_{F}-\frac{k_{g}}{c_{p} L e_{F}} \frac{\partial Y_{F}}{\partial y}\right\}_{0^{+}}=\dot{m}_{s}(z)$,

$\left\{\dot{m}_{s}(z) Y_{O}-\frac{k_{g}}{c_{P} L e_{O}} \frac{\partial Y_{O}}{\partial y}\right\}_{0^{+}}=0$

$\left\{k_{g} \frac{\partial T}{\partial y}\right\}_{0^{+}}-\left\{k_{s} \frac{\partial T}{\partial y}\right\}_{0^{-}}=\dot{m}_{s}(z) L_{v}$

$$
+\epsilon \sigma\left(T_{s}^{4}(z)-T_{\infty}^{4}\right),
$$

where $k_{s}$ is the solid-phase heat conductivity, $L_{v}$ is the heat of surface pyrolysis per unit mass of fuel, $\sigma$ is the Stefan-Boltzmann constant, and $\epsilon$ the surface emissivity. In Eq. 15 we have replaced $(V-\dot{r})$ by $V$ because the interface regression velocity, $\dot{r}=\dot{m}_{s} / \rho_{s}$, is small compared with the gas-phase value $V_{0^{+}}$, due to the small value of the ratio between the gas and solid densities. The interface regression velocity $\dot{r}$ leads to changes with $z$ of the cylinder radius, given by $d a / d z=-\dot{r} / W_{f}$, which we also neglect in the flame spread region.

We look for a solution of the previous system of equations representing an ignition flame front that separates the nonburning region, downstreams at $z \rightarrow \infty$, and the upstream, $z \rightarrow$ $-\infty$, burning region. Downwind the flow variables must approach

$\left.\begin{array}{l}A \rightarrow \hat{A}(y), W \rightarrow \hat{W}(y), \\ Y_{O} \rightarrow Y_{O \infty}, Y_{F} \rightarrow 0, T \rightarrow T_{\infty}\end{array}\right\}$ for $z \rightarrow+\infty$

Here, the functions $\hat{A}(y)$ and $\hat{W}(y)$ are given by the nonburning, no-vaporization solution of the previous system of equations with $T_{s}=T_{\infty}$, justified only for large activation energies of the pyrolysis reaction.
Upstream, the solution must approach, asymptotically, the one-dimensional steady burning solution of the system of equations (3-18), with $\partial / \partial z=0$,

$$
\begin{aligned}
& \left.\begin{array}{l}
A \rightarrow \tilde{A}(y), W \rightarrow \tilde{W}(y), \\
Y_{O} \rightarrow \tilde{Y}_{O}(y), Y_{F} \rightarrow \tilde{Y}_{F}(y), T \rightarrow \tilde{T}(y)
\end{array}\right\} \\
& \qquad \text { for } z \rightarrow-\infty .
\end{aligned}
$$

In the region $z \rightarrow-\infty$, the energy equation (11) can be readily integrated in the solid to give the interface condition

$$
\frac{\left(\dot{m}_{s}\right)_{z \rightarrow-\infty}}{\rho_{s}}\left(T_{v}-T_{\infty}\right)=\alpha_{s}\left\{\frac{\partial T}{\partial y}\right\}_{0^{-}} .
$$

Finally, notice that the pyrolysis rate $\dot{m}_{s}(z)$ (unknown for $z \leq 0$, and zero for $z>0$ ) and the surface temperature $T_{s}(z)$ (equal to $T_{v}$ for $z \leq 0$, and unknown for $z>0$ ) must be obtained, as part of the solution, together with the flame spread velocity $W_{f}$, an eigenvalue of the problem.

\section{ORDER OF MAGNITUDE ESTIMATES}

We shall write the previous system of equations in nondimensional form, using as scales appropriate characteristic lengths and velocities for the flame spread region. These scales, as well as the characteristic value of the flame spread velocity $W_{f c}$, will be obtained, together with some preliminary physical insight into the problem, by means of order of magnitude estimates of the terms appearing in the system of equations given in the previous section.

Let $\ell$ be the characteristic length in the $z$ direction of the flame front region (equal for the solid and gas phases) and $\delta_{g}$ and $\delta_{s}$ the characteristics thickness of the gas-phase boundary layer and of the heated layer in the solid. Let $W_{\infty}$ and $V_{c}$ be the characteristic values of the velocity components longitudinal and transverse to the boundary layer, respectively, and let the strain rate be of order $A_{e}$. From the continuity equation, $A_{e} \approx V_{c} / \delta_{g} \approx W_{c o} / \ell$ we obtain

$\ell / a \approx W_{\infty} / v_{\infty} \approx 1$,

because we analyze the distinguished case where $w_{\infty}$ and $v_{\infty}$ are of the same order. 
The thickness of the gas-phase boundary layer is obtained from the requirement that the convective and diffusive terms in the momentum equation for $A$ must be of the same order, i.e., $A_{e}^{2} \approx \alpha_{\infty} A_{e} / \delta_{g}^{2}$, where $\alpha_{\infty}=k_{\infty} / \rho_{\infty} c_{p}$ is the air thermal diffusivity. Then the boundary layer thickness $\delta_{g}$ is given by

$\delta_{g} \approx \sqrt{\alpha_{\infty} / A_{e}}$, or $\delta_{g} \approx a / \sqrt{R e_{c}}$,

where $R e_{c}=a^{2} A_{e} / \alpha_{\infty}=\kappa a V_{\infty} / \alpha_{\infty}$ is the characteristic transverse Peclet, or Reynolds number. The characteristic value, within the boundary layer, of the velocity transverse to the layer is $V_{c}=\sqrt{\alpha_{c o} A_{e}}$.

From the energy balance at the interface $k_{\infty}\left(T_{f}-T_{v}\right) / \delta_{g} \approx k_{s}\left(T_{v}-T_{\infty}\right) / \delta_{s}$, where

$$
\begin{aligned}
\frac{T_{f}}{T_{\infty}}= & {\left[\frac{q_{F}}{c_{p} T_{\infty}} Y_{O_{\infty}}+s-\left(\frac{c_{s}}{c_{p}}\left(\frac{T_{p}}{T_{\infty}}-1\right)+\frac{L_{v}}{c_{p} T_{\infty}}\right.\right.} \\
& \left.\left.-\frac{T_{v}}{T_{\infty}}\right)\right] /\left(s+Y_{O \infty}\right)
\end{aligned}
$$

is the flame temperature in the upstream burning region, in the Burke-Schumann limit of infinite gas-phase reaction rates and in absence of radiation losses; this will be used in the following as a reference flame temperature. Then, the ratio of characteristic thickness of the gas boundary layer and solid heated layer is

$\frac{\delta_{g}}{\delta_{s}} \approx \frac{k_{\infty}}{k_{s}} \frac{T_{f}-T_{v}}{T_{v}-T_{\infty}}=N$

where, the factor $N$ is of order unity for most plastic fuels.

Finally one relation between the characteristic flame front velocity, $W_{f c}$, and the thickness of the solid thermal layer is obtained from solid energy equation

$W_{f c} \frac{T_{v}-T_{\infty}}{\ell} \approx \alpha_{s} \frac{T_{v}-T_{\infty}}{\delta_{s}^{2}}$.

Then, $W_{f c} \approx \alpha_{s} \ell / \delta_{s}^{2}$, and we obtain, for the characteristic flame spread velocity

$W_{f c} \approx W_{\infty} \frac{\alpha_{s}}{\alpha_{\infty}} N^{2}$.

Notice that this estimate for the flame spread rate was also obtained by de Ris [7] for flame spread rate in opposed flow with velocity $W_{\infty}$ in the flame front region. Although this dependence with the longitudinal velocity of the spread rate is the same in both cases, it is important to point out that the flame structure and its dependence on $W_{\infty}$ is very different. When the longitudinal velocity increases, the flame spread increases because the flame front region becomes longer; whereas in the opposed flame spread configuration, the thickness of the boundary layer in the gas phase decreases as the longitudinal velocity increases, and then the heat flux from the gas increases because the flame approaches to the surface. Thus, with increased flow velocities, in downwind propagation, the flame front is longer, whereas in the case of up-wind propagation the flame is closer to the surface.

Observe that the assumption $W_{f} \ll W_{\infty}$, made in the previous section, is valid if $\alpha_{s} \ll$ $\alpha_{\infty}$, which is the case for most plastic fuels. Notice also that if $N \approx 1, \delta_{g} \approx \delta_{s}$ is small compared with $a$ if $R e_{c} \gg 1$.

Finally, the regression velocity $\dot{r}$ whose order is $k_{\infty}\left(T_{f}-T_{v}\right) / \delta_{g} \rho_{s} L_{v}$ according to Eq. 18 can be compared with $W_{f c}$ to give $\dot{r} / W_{f c} \approx c_{s}\left(T_{v}-\right.$ $\left.T_{\infty}\right) / L_{v} N \sqrt{R e_{c}}$, which for large values of $R e_{c}$ is small compared with unity justifying the previous assumptions.

\section{NONDIMENSIONAL EQUATIONS}

To write the equations in nondimensional form, we measure $A$ with $A_{e}, W$ with $W_{c}, V$ with $V_{c}$, $z$ with $\ell$, and the coordinate $y$ with $\delta_{g}$ in the gas phase and with $\delta_{g} k_{s} / k_{\infty}$ in the solid phase. The oxygen mass fraction, the density, and the temperature will be measured with their ambient values $Y_{O \infty}, \rho_{\infty}$, and $T_{\infty}$.

We thus obtain the following nondimensional system of boundary layer equations for the gas phase $(y>0)$,

$$
\begin{aligned}
& \rho A+\frac{\partial(\rho V)}{\partial y}+\frac{\partial(\rho W)}{\partial z}=0, \\
& \rho A^{2}+\rho V \frac{\partial A}{\partial y}+\rho W \frac{\partial A}{\partial z}=1+\operatorname{Pr} \frac{\partial}{\partial y}\left(T^{n} \frac{\partial A}{\partial y}\right),
\end{aligned}
$$

$$
\rho V \frac{\partial W}{\partial y}+\rho W \frac{\partial W}{\partial z}=\operatorname{Pr} \frac{\partial}{\partial y}\left(T^{n} \frac{\partial W}{\partial y}\right),
$$


$\rho V \frac{\partial \beta_{1,2}}{\partial y}+\rho W \frac{\partial \beta_{1,2}}{\partial z}=\frac{\partial}{\partial y}\left(T^{n} \frac{\partial \beta_{1,2}}{\partial y}\right)$,

$\rho V \frac{\partial T}{\partial y}+\rho W \frac{\partial T}{\partial z}=\frac{\partial}{\partial y}\left(T^{n} \frac{\partial T}{\partial y}\right)-\dot{\omega}$.

The two equations for the mass fractions, $Y_{F}$ and $Y_{O}$, are replaced by two equations for the following coupling functions

$\beta_{1}=Y_{O}-S Y_{F}$ and $\beta_{2}=(1-\Lambda) Y_{O}+\Lambda T$,

where $S=s / Y_{O \infty}$ is the overall mass stoichiometric ratio, $\Lambda=S /(S+q)$ and $q=q_{F} / c_{p} T_{\infty}$ is the nondimensional heat of reaction, and $L_{O}=L_{F}=1$.

The nondimensional reaction term is given by

$\dot{\omega}=-\rho D Y_{F} Y_{O} e^{T_{a} / T}$,

where $T_{a}=E_{g} / R T_{\infty}$ is the nondimensional activation temperature, and

$D=\frac{q_{F}}{c_{p} T_{\infty}} \frac{Y_{O_{\infty}} B_{g}}{A_{e}}=\frac{q_{F}}{c_{p} T_{\infty}} \frac{Y_{O \infty}}{\operatorname{Re}_{c}} \frac{B_{g} a^{2}}{\alpha_{\infty}}$,

is the nondimensional Damköhler number.

The energy equation for the solid phase takes the form

$\frac{\dot{\mu}_{s}}{\chi} \frac{\partial T}{\partial y}-\Omega \frac{\partial T}{\partial z}=\frac{\partial^{2} T}{\partial y^{2}}$,

where $\dot{\mu}_{s}=\dot{m}_{s} / \rho_{\infty} \sqrt{\alpha_{\infty} U_{e}}$ is the nondimensional surface gasification rate, $\chi=c_{p} / c_{s}$ is the gas to solid ratio of specific heats at constant pressure, and

$\Omega=W_{f} /\left\{W_{\infty} \frac{\alpha_{s}}{\alpha_{\infty}}\left(\frac{k_{\infty}}{k_{s}}\right)^{2}\right\}$

is the nondimensional spread rate.

The boundary conditions take the form

$y \rightarrow+\infty: A, W, \beta_{1}, \beta_{2}$, and $T \rightarrow 1$,

$y \rightarrow-\infty: T \rightarrow 1$,

$z \rightarrow-\infty: A \rightarrow \tilde{A}(y), W \rightarrow \tilde{W}(y)$,

$\beta_{1} \rightarrow \tilde{\beta}_{1}(y), \beta_{2} \rightarrow \tilde{\beta}_{2}(y), T \rightarrow \tilde{T}(y)$,

$z \rightarrow+\infty: A \rightarrow \hat{A}(y), W \rightarrow \hat{W}(y)$,

$\beta_{1} \rightarrow 1, \beta_{2} \rightarrow 0, T \rightarrow 1$.
And, finally, at the solid surface the boundary conditions are

$\{\rho V\}_{0^{+}}=\dot{\mu}_{s}$,
$A_{0^{+}}=W_{0^{+}}=0$,

$T_{0^{+}}=T_{0^{-}}=T_{s}(z)$,$$
\left\{\dot{\mu}_{s} \beta_{1}-T^{n} \frac{\partial \beta_{1}}{\partial y}\right\}_{0^{+}}=-S \dot{\mu}_{s},
$$

$$
\begin{aligned}
\left\{\dot{\mu}_{s} \beta_{2}-T^{n} \frac{\partial \beta_{2}}{\partial y}\right\}_{0^{+}}= & -\Lambda\left[q_{s}(z)+\dot{\mu}_{s}\left(\lambda-T_{s}\right)\right. \\
& \left.+S_{R}\left(T_{s}^{4}-1\right)\right]
\end{aligned}
$$

$q_{g}(z)-q_{s}(z)=\lambda \dot{\mu}_{s}+S_{R}\left(T_{s}^{4}-1\right)$,

where $\lambda=L_{v} / c_{p} T_{\infty}$ is the nondimensional heat of pyrolysis, and $q_{g}(z)=\left\{T^{n} \partial T / \partial y\right\}_{0^{+}}$and $q_{s}(z)=\{\partial T / \partial y\}_{0^{-}}$are the nondimensional heat fluxes from the gas and to the solid, respectively. In addition, the surface temperature must be constant, $T_{s}(z)=T_{v}$, for $z \leq 0$, and the solid gasification rate must be zero, $\dot{\mu}_{s}(z)=0$, at $z>0$.

The radiation parameter

$$
S_{R}=\frac{\epsilon \sigma T_{\infty}^{3}}{k_{\infty}} \frac{a}{\sqrt{R e_{c}}},
$$

similar in form to the one used by Bhattacharjee and Altenkirch [17], measures the ratio between the radiant heat losses and the heat conducted to the solid.

The problem is to calculate, as an eigenvalue, the nondimensional spread rate $\Omega$, as well as the surface distribution of temperature and pyrolysis mass flux. The solution involves, among other parameters, the mass stoichiometry ratio $S$, inversely proportional to the oxygen concentration, the nondimensional Damköhler number $D$, characterizing the kinetics, and the radiation loss parameter $S_{R}$.

\section{NUMERICAL ANALYSIS}

\section{Basic Procedure}

For positive values of $W_{\infty}$ the gas-phase value of $W$ is positive. Then, the gas-phase boundary 
layer equations are parabolic with information convected in the positive $z$ direction; so they must be integrated marching in the positive $z$ direction beginning with the selfsimilar twodimensional burning solution at $z \rightarrow-\infty$. On the other hand, the solid energy equation is also parabolic, but it must be integrated marching in the negative $z$ direction. This opposite parabolic characteristic of the model led us to use an iterative procedure, integrating the gas and solid equations sequentially.

If, for the moment, we consider the distribution of heat flux toward the interior of the solid, $q_{s}(z)$, as known for $z<0$ and the surface temperature, $T_{s}(z)$, known for $z>0$, we can integrate the gas conservation equations for $z<$ 0 , using the assumed $q_{s}(z)$ and constant surface temperature $T_{v}$, allowing us to obtain the mass flow rate $\dot{\mu}_{s}(z)$ from the energy balance at the surface. The integration is continued for $z>0$ in terms of the assumed $T_{s}(z)$ and calculating, using the energy balance at the surface, the heat flux, $q_{g}(z)$, from the gas. Then, we integrate the solid energy equation, marching in the negative $z$ direction from $z \rightarrow+\infty$, using the distribution $q_{s}(z)=q_{g}(z)$ obtained from the gas phase. The flame spread rate $\Omega$ that enters in the solid energy equation must be calculated iteratively, so as to reach the pyrolysis temperature $T_{v}$ at $z=y=0$. Then, with this calculated value of $\Omega$, the integration of the solid energy equation is continued to the negative values of $z$, using the condition of constant surface temperature $T_{v}$ and the mass flow gasification rate, $\dot{\mu}_{s}(z)$, obtained previously in the gas-phase calculations, thus generating the new estimate of $q_{s}(z)$.

The sequential integration of the gas and solid equations gives the spread rate in a few iterations (usually $5-10$ iterations to reduce the error in $\Omega$ to less than $0.1 \%$ ).

\section{Numerical Algorithm}

A modified version of the Box Method of Keller $[15,16]$, an implicit scheme, is used to integrate the two systems of nonlinear parabolic differential equations. The method provides a discretization in tridiagonal matrix form; the nonlinear terms have been treated by a simple iterative update of the matrix coefficients coupled with a linearization of the reaction term.
One of the main features of the Box Method is the possibility of using, with second-order accuracy, a nonuniform mesh in the crosswise coordinate. For large values of the Damköhler number the flame thickness becomes very thin, so that it is necessary to place a large number of points in the flame region to decrease the numerical error due to the exponential reaction term. To solve this problem accurately enough, with the least computing time, we have used an adaptive mesh in the flow field, reconstructing the mesh periodically, using the error measure proposed by Dwyer et al. [18]. In this way it has been possible to cover the wide range of Damköhler numbers, from high enough when the flame is very thin and the Burke-Schumann approximation of infinite chemical kinetics is applicable, down to the smaller values leading to extinction.

\section{RESULTS AND DISCUSSION}

The flame spread rate calculations have been carried out with physical constants corresponding to polymethylmethacrylate (PMMA) and ambient gas properties of standard air (Table 1). We thus obtain the following nondimensional physical constants:

$$
\begin{aligned}
T_{v} & =2.3, T_{a} \\
& =75.2, q \\
& =80.1, \lambda \\
& =4.9, \text { and } \chi \\
& =0.6 .
\end{aligned}
$$

Three values of the oxygen mass fractions have been considered: near PMMA flammability limit $\left(Y_{O \infty}=0.18, S=10.67\right)$, normal atmosphere $\left(Y_{O \infty}=0.23, S=8.35\right)$, and high oxygen content $\left(Y_{O \infty}=0.33, S=5.82\right)$ although not enough to originate dissociation and thus invalidate the irreversible global reaction approach. The calculations have been made for a wide range of the Damköhler number, $D$, and two values of the emissivity coefficient $(\epsilon=$ 0 and 1).

We began with the Burke-Schumann analyses of infinitely fast chemical kinetics (BS), which 
TABLE 1

Data Used for Computation ${ }^{a}$

\begin{tabular}{lll}
\hline Symbol & \multicolumn{1}{c}{ Value } & \multicolumn{1}{c}{ Units } \\
\hline$E_{g}$ & 180,030 & $\mathrm{~J} / \mathrm{mol}$ \\
$B_{g}$ & $2.5 \cdot 10^{9}$ & $\mathrm{~s}^{-1}$ \\
$q_{F}$ & $2.5952 \cdot 10^{7}$ & $\mathrm{~J} / \mathrm{kg}$ (PMMA) \\
$s$ & 1.92 & \\
$\rho_{s}$ & 1200 & $\mathrm{~kg} / \mathrm{m}^{3}$ \\
$k_{s}$ & 0.225 & $\mathrm{~J} / \mathrm{m}^{\circ} \mathrm{Ks}$ \\
$c_{s}$ & 1875 & $\mathrm{~J} / \mathrm{kg}^{\circ} \mathrm{K}$ \\
$L_{v}$ & $1.5884 \cdot 10^{6}$ & $\mathrm{~J} / \mathrm{kg}^{\circ}(\mathrm{PMMA})$ \\
$T_{v}$ & 663 & ${ }^{\circ} \mathrm{K}$ \\
$T_{\infty}$ & 288 & ${ }^{\circ} \mathrm{K}$ \\
$c_{p}$ & 1125 & $\mathrm{~J} / \mathrm{kg}^{\circ} \mathrm{K}$ \\
$k_{\infty}$ & 0.02524 & $\mathrm{~J} / \mathrm{m}^{\circ} \mathrm{Ks}$ \\
$\alpha_{\infty}$ & $1.8323 \cdot 10^{-5}$ & $\mathrm{~m}^{2} / \mathrm{s}$ \\
$P r$ & 0.73 & \\
$n$ & 0.8 & \\
\end{tabular}

${ }^{a}$ The thermochemical data of the PMMA have been chosen from Ref. 3.

already allow us to describe the main fluiddynamic characteristics of the spread mechanism. Moreover, it constitutes the appropriate reference solution in order to evaluate the influence of the finite chemical kinetic effects. These effects are analyzed for values of the Damköhler number ranging from high enough, where the results tend to the BS results, to sufficient low Damköhler numbers, where extinction occurs. Finally, some results are presented taking into account the influence of radiation.

\section{Flame Structure with Infinite Reaction Rates}

In the Burke Schumann limit (BS) of high Damköhler number, reactants only coexist in a very thin reaction layer with very small values of the concentrations. Thus the energy equation simplifies to the Burke-Schumann equilibrium relation $Y_{O} Y_{F}=0$, implying that the reactants do not coexist, and we can obtain the temperature and concentrations in terms of the coupling functions $\beta_{1}$ and $\beta_{2}$ by

$$
\begin{aligned}
Y_{O} & =0, Y_{F} \\
& =-\beta_{1} / S, T \\
& =\left(\beta_{2}-(1-\Lambda) \beta_{1}\right) / \Lambda \text { when } \beta_{1} \leq 0 \\
Y_{F} & =0, Y_{O}=\beta_{1}, T=\beta_{2} / \Lambda \text { when } \beta_{1} \geq 0
\end{aligned}
$$
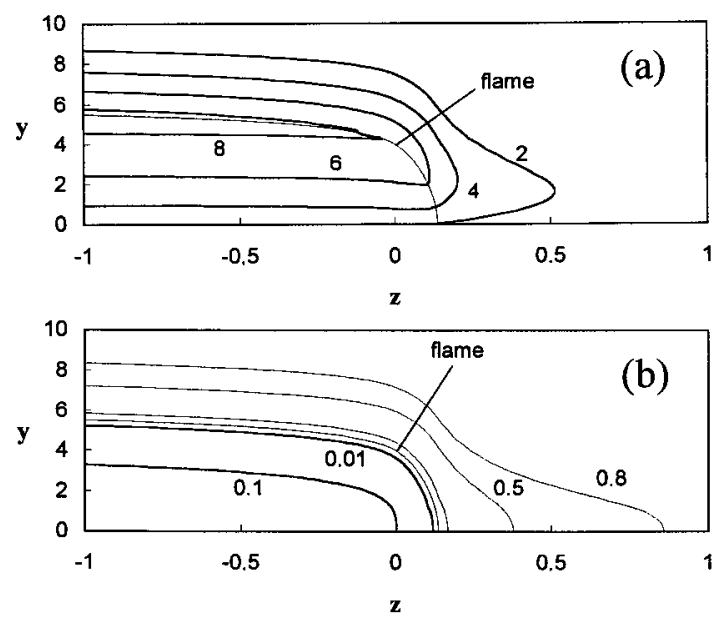

Fig. 2. Nondimensional contours levels in the Burke-Schumann approximation for $Y_{O \%}=0.23$ and $\epsilon=0$. (a) Temperature levels. (b) Contours of oxygen mass fraction, $Y_{O} / Y_{O \infty}$, in thin traces and fuel mass fraction, $Y_{F}$, in thick ones. The calculated flame spread velocity is, in this case, $\Omega_{B S}=9.13$

In Fig. 2, we give details of the BS flame structure, for the ambient oxygen mass fraction of 0.23 , with the surface emissivity factor $\epsilon$ set equal zero. The nondimensional isotherm map is presented in Fig. 2a, where the flame position, defined by $\beta_{1}=0$, is represented with fine trace. The fuel and oxygen mass fraction contour levels are represented in Fig. 2b. It can be seen that the diffusion flame remains nearly parallel to the wall in the negative side of the $z$ axis, and then falls down to the solid surface in the domain $z>0$ where there is no gasification. The flame reaches the surface with an angle of $90^{\circ}$, imposed by the boundary condition of zero pyrolysis mass flux at $z>0$. This displacement of the flame ahead of the pyrolysis front is due to the longitudinal convective flow, which also aids in the transport of heat from the gas to the solid surface. As a consequence of the longitudinal convective flow the isotherms shown in Fig. 2 a have a characteristic nose shape, and the heat flux to the solid (see Fig. 5) reaches a maximum in the point where the flame touches the surface.

\section{Flame Structure with Finite Reaction Rates}

The flame structure with finite reaction rates is shown in Fig. 3, for an ambient oxygen mass 

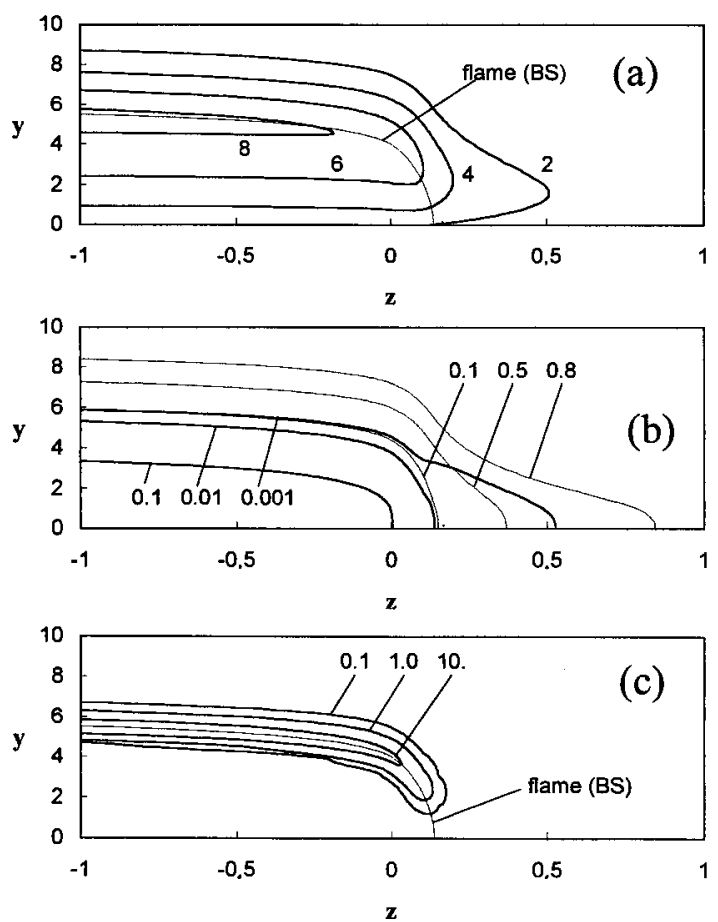

Fig. 3. Nondimensional contours at a Damköhler number of $D=10^{10}$ with $Y_{O x}=0.23$ and $\epsilon=0$. (a) Temperature levels. (b) Contours of oxygen mass fraction, $Y_{O} / Y_{O}$, in thin traces and fuel mass fraction, $Y_{F}$, in thick ones. (c) The contour level of production term, $\dot{\omega}$. The calculated flame spread velocity is, in this case, $\Omega=8.51$.

fraction of 0.23 , a value of the Damköhler number $10^{10}$, and $\epsilon=0$. The nondimensional isotherms are presented in Fig. 3a, while the mass fraction contour levels for the fuel and oxygen are represented in Fig. 3b, and the nondimensional reaction term in Fig. 3c. For comparison, the BS flame position, as given for the same conditions in the infinite Damköhler number limit, is represented in fine trace.

In this finite Damköhler number case, the flame may be expected to quench before reaching the wall, due to a lowering of the flame temperature. When the flame approaches the wall we begin to see, as shown in Fig. 3b, increasing amounts of fuel leaking through the reaction zone. The oxygen, in smaller quantities, also crosses the reaction zone but the effect is less visible since, due to the flame configuration and the gas flow pattern, the oxygen has to move across the reaction zone by traverse diffusion. There is a frozen region near the wall, where fuel and oxygen coexist, while the reactants are
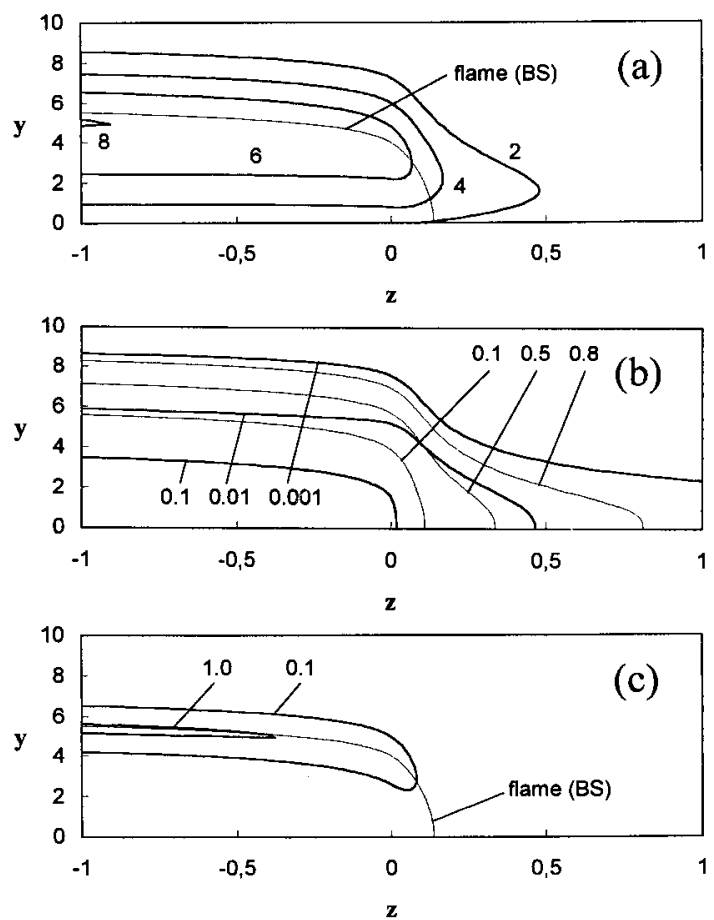

Fig. 4. Nondimensional contours at a Damköhler number of $D=10^{9}$ with $Y_{O \propto}=0.23$ and $\epsilon=0$. (a) Temperature levels. (b) Contours of oxygen mass fraction, $Y_{O} / Y_{O \infty}$, in thin traces, and fuel mass fraction, $Y_{F}$, in thick ones. (c) The production term, $\dot{\omega}$. The calculated flame spread velocity is, in this case, $\Omega=6.95$.

convected by the longitudinal velocity component $W$ downwind of the flame, while moving outwards from the symmetrical plane by azimuthal convection. For this large value of $D$, $10^{10}$, the flame shape and location (see Fig. 3c) is well described by the BS solution in most of the flow field, although not near the surface where, due to the lower temperatures, the reaction is quenched.

In Fig. 4 the flame structure is shown for a Damköhler number $D=10^{9}$, lower than the previous by an order of magnitude. The size of the premixed region and the fuel leakage are seen to increase with decreasing values of the Damköhler number. In addition, the leading edge of the flame moves upstream along the mixture fraction line corresponding to the Burke-Schumann solution.

Figure 5 shows, for various values of the Damköhler number, the variation along the fuel rod surface of the heat flux from the gas phase to the rod. This flux reaches its maximum value 


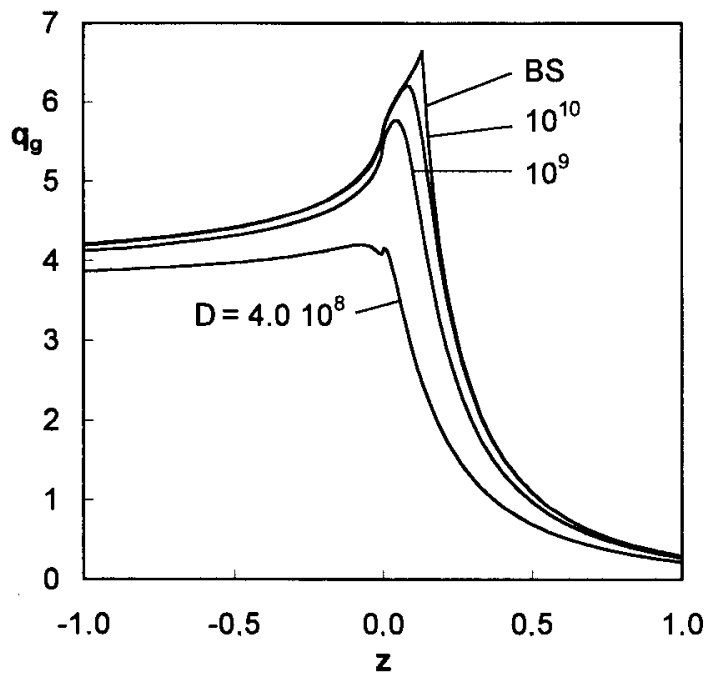

Fig. 5. Nondimensional heat flux from the gas to the solid for different Damköhler numbers ( $Y_{O x}=0.23, \epsilon=0$ ).

in the flame edge region of the front. In the BS limit, $q_{g}$ has its maximum value at the point where the flame touches the surface. With decreasing values of $D$ the peak becomes smoother and closer to the origin of the vaporization line; with the decreasing values of the heat flux to the solid the spread rate falls down.

We show, in Fig. 6, how radiation from the surface of the rod affects the flame structure. The calculations were carried out for $\epsilon=1$, $Y_{O \varkappa}=0.23$, and $D=10^{9}$ to compare with the case $\epsilon=0$ shown in Fig. 4. The main effects are an expected lowering of the temperatures and a significant increase in the size of the premixed region. The extent of the region of nondimensional reaction rate larger than 0.1 does not change much, because the effect of the lowering temperature is compensated by an increase in the concentration of the mixed reactants. The flame approaches the rod and its leading edge moves upstream, farther from the vaporization region.

\section{Flame Spread Rate}

The results for the flame spread rate are presented, for convenience, in the form of a reduced nondimensional spread velocity, $\bar{\Omega}=$ $\Omega / \Omega_{B S}$, versus a modified Damköhler number $\bar{D}$, based on a more accurate estimate of the characteristic chemical time. To obtain this, we
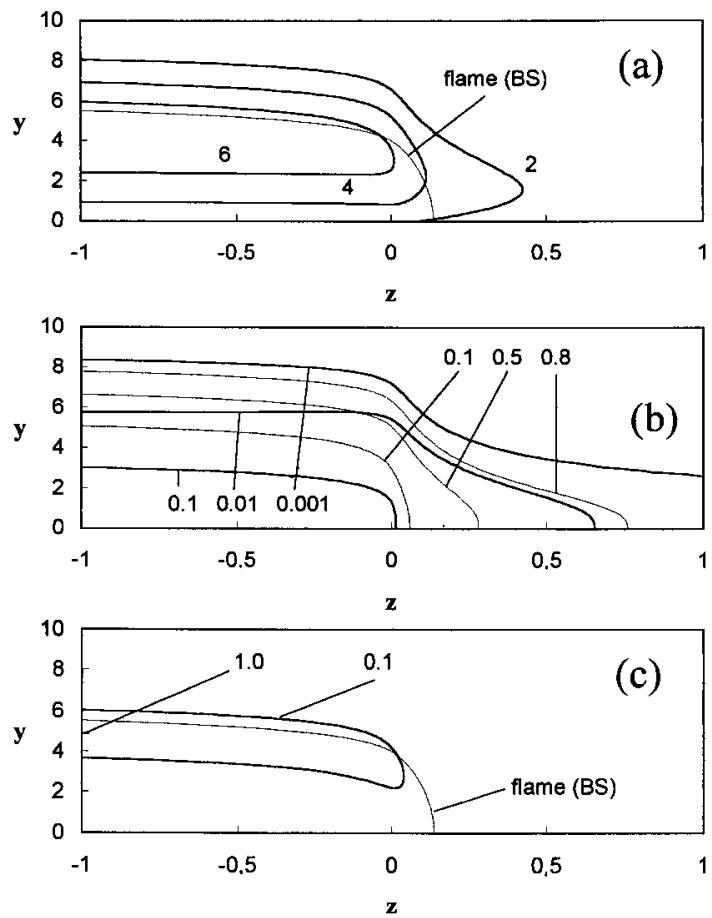

Fig. 6. Nondimensional contours at a Damköhler number of $D=10^{\circ}$ with $Y_{O \propto}=0.23$ and $\epsilon=1$. (a) Temperature levels. (b) Contours of oxygen mass fraction, $Y_{O} / Y_{O \infty}$, in thin traces and fuel mass fraction, $Y_{F}$, in thick ones. (c) The production term, $\dot{\omega}$. The calculated flame spread velocity is, in this case, $\Omega=3.58$.

begin by noticing that large activation energy asymptotics leads to the following relations:

$U_{L}^{2}=\frac{4 B_{g} \alpha_{\rho} \rho_{f} Y_{O \infty}}{\rho_{\infty}}\left(\frac{s}{Y_{O \infty}} \frac{C_{p} T_{\infty}}{q_{F}} \frac{T_{\infty}}{T_{a}}\right)^{3} e^{-T_{a} / T_{f}}$,

for $U_{L}$, the laminar burning velocity of a stoichiometric premixed flame, in terms of the dimensional variables. From this, it is possible to calculate the residence time in the flame, $\alpha_{f} / U_{L}$, which together with the residence time, $A_{e}^{-1}$, defines the Damköhler number, $U_{L}^{2} / \alpha_{f} A_{e}$. This is roughly equal to the modified Damköhler number given by

$\bar{D}=\frac{4}{T_{f} q}\left(\frac{S T_{f}^{2}}{q T_{a}}\right)^{3} D e^{-T_{u} / T_{f}}$.

to be used below in the description of kinetic effects on flame spread.

When, in Fig. 7, we now plot the reduced spread rate, $\bar{\Omega}=\Omega / \Omega_{B S}$, in terms of $\bar{D}$, we 


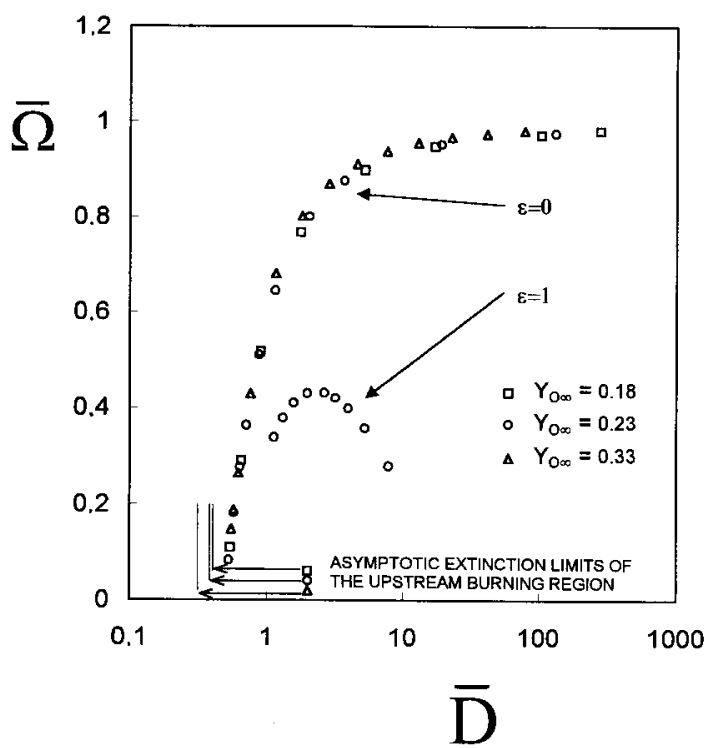

Fig. 7. The reduction of the flame spread velocity due to finite rate effects, represented by the corrected nondimensional spread velocity, $\bar{\Omega}=\Omega / \Omega_{B S}$, as a function of the modified Damköhler number $\bar{D}$ defined in Eq. 50, where $\Omega_{B S}$ is equal $5.77,9.13$, and 16.7 for $Y_{O \propto}$ equal to $0.18,0.23$, and 0.33 , respectively.

account for the major influence of finite chemical effects. In this figure we show the calculated values for three oxygen mass fraction $\left(Y_{0 \times}=\right.$ $0.18,0.23$, and 0.33 ) without effects of the radiation losses $(\epsilon=0)$, and for $Y_{O \infty}=0.23$ with $\epsilon=1$. The calculated values for $\epsilon=0$ nearly collapse into a single curve.

For large Damköhler numbers or low strain rates, the reaction is very fast and the effects of finite gas-phase chemical kinetics become unimportant. The heat transfer from the gas to the solid controls the spread process, and the spread rate tends asymptotically to the BS value $\bar{\Omega}=1$. For large strain rates, the reaction is slow and chemical kinetics becomes important, and the spread rate depends strongly of the strain rate. In this case, finite rate chemical kinetic effects control the spread mechanism and the spread rate decrease monotonously, until extinction occurs.

The flame structure in the upstream region, where the two-dimensional configuration is independent of $z$, is also affected by the lowering of $\bar{D}$. The vertical boundaries of Fig. 7 represent the extinction limits for the counter flow flame at $z \rightarrow-\infty$, calculated with the asymptotic theory of Liñan [19]. The results of our calculations show that the spread process stops for Damköhler numbers larger, although moderately close to the extinction values of the twodimensional flame. Nevertheless, we consider that this phenomenon has not been investigated in depth in this work, because our numerical scheme leads to instabilities in the region of very low nondimensional spread rates.

The effect of surface radiation losses on the flame spread rate can be seen in Fig. 7. The spread process is dominated at low $\bar{D}$ by finite chemical rate effects, and heat losses by radiation become negligible. When $\bar{D}$ increases, the effects of finite chemical kinetics become unimportant, but the flame spread process begins to be influenced by surface radiation losses, so that a maximum flame spread velocity appears in the $\bar{\Omega}(\bar{D})$ curve. Thus, for appropriately large values of $\bar{D}$, radiation losses will eventually affect the spread process, and the spread velocity will decrease monotonously until extinction occurs. This is so because when the heat emitted by radiation by the solid surface in the vaporization region, $\epsilon \sigma T_{v}^{4}$, becomes of the order of the heat reaching the surface by conduction, $k_{\infty}\left(T_{f}-\right.$ $\left.T_{v}\right) / \delta_{g}$, the fuel vaporization flux and, then, the flame spreading velocity is significantly reduced. The ratio of the two heat fluxes $\bar{S}_{R}=\dot{\epsilon} \sigma T_{\nu}^{4}\left(\alpha_{x}\right)$ $\left.A_{e}\right)^{1 / 2} / k_{x}\left(T_{f}-T_{v}\right)$, grows like $\bar{D}^{1 / 2}$, with decreasing values of the strain rate, $A_{e}=\kappa v_{x} / a$. $\bar{S}_{R}$ is more appropriate to measure the radiation losses than the parameter $S_{R}$ introduced in the formulation.

For values of this ratio above a critical value, of order unity, the flame structure will not survive in the upstream burning region, as shown by T'ien [13]. We find that the flame spread process is no longer possible for smaller values of this ratio, still of order unity. When with decreasing values of $v_{\infty}, \bar{S}_{R}$ grows to values of order unity, the fraction of the heat coming from the gas phase that not is lost by radiation and is not used for vaporization, may not be enough to raise the temperature of the solid from $T_{\infty}$ to $T_{v}$ and, then, flame spread is no longer possible.

The reduction in flame spread velocity due to the effect of surface radiation is shown in Fig. 8 in the Burke-Schumann limit, $\bar{D} \rightarrow \infty$, of infinite gas-phase reaction rates. The limiting 


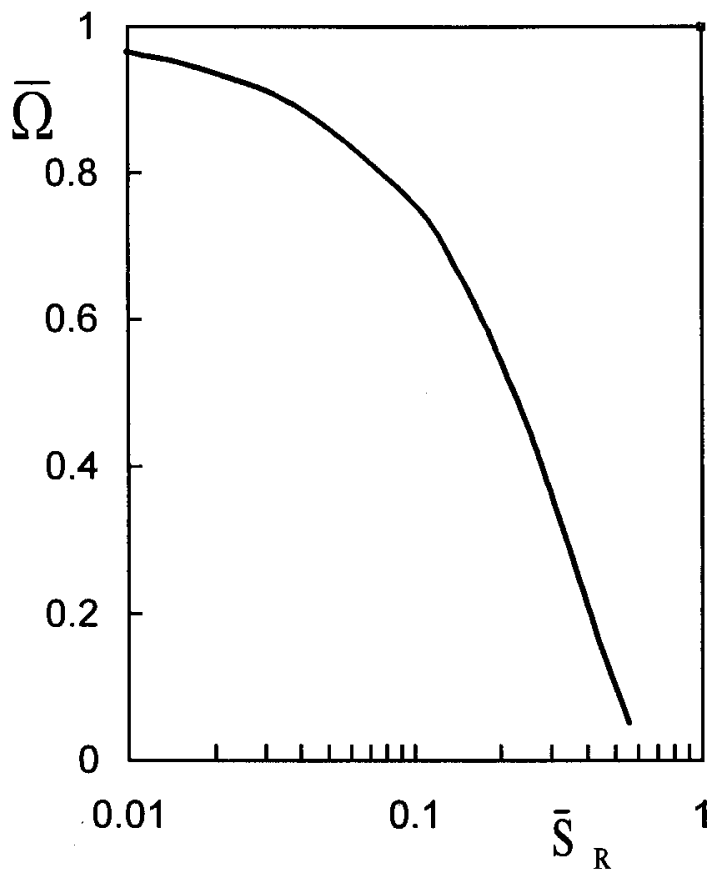

Fig. 8. The reduction in flame spread velocity, $\bar{\Omega}=\Omega / \Omega_{B S}$, as function of the radiation parameter $\bar{S}_{R}$ in the BurkeSchumann limit of infinite gas-phase reaction rates $\left(Y_{O \infty}=\right.$ $0.23, \epsilon=1$ ).

value of $\bar{S}_{R}$ from flame spread to occur is roughly $\bar{S}_{R}=0.6$ for $Y_{O \infty}=0.23$.

\section{CONCLUSIONS}

The wind-aided flame spread process along a solid fuel rod under an oblique forced flow, a generic case not considered previously in the literature, has been described for values of the transverse flow velocity component large enough that $v_{x} a / \alpha_{x} \gg 1$, and we can use the boundary layer approximation in its description. In the analysis we include the effects of finite rate of the gas-phase reaction, modeled as a global second-order Arrhenius reaction, and also the effects of radiant losses from the surface. We have not included the effects of radiation from the gas phase, because in this configuration, and for large Reynolds numbers of the transverse velocity, the heat transfer by convection is typically dominant. The pyrolysis law of the fuel is considered to have a strong dependence on the surface temperature, so that fuel gasification only occurs when $T_{s}$ reaches a temperature $T_{v}$. Gravity effects have been left out of the analysis based on the assumption that $g a / v_{\infty}^{2} \ll 1$.

The numerical results show the detailed structure of the reacting flow field, and allow us to calculate the flame spread velocity as long as it is positive. This velocity is given in order of magnitude by Eq. 27, so that it is proportional to the longitudinal component of the forced flow velocity. The nondimensional spread velocity, defined in Eq. 37, is in the Burke-Schumann approximation mainly dependent on the oxygen mass fraction of the environment. Due to finite rate effects and radiation losses, the flame spread is reduced by a factor $\bar{\Omega}=\Omega / \Omega_{B S}$. If we leave out of the analysis the effects of surface radiation, the spread rate occurs at high Damköhler numbers in a diffusion-controlled form, well described by the Burke-Schumann approximation; when the Damköhler number decreases the spread rate decreases until flame spread is no longer possible at a Damköhler number $\bar{D}$, of order unity, higher than the extinction value of the upstream cylindrical flame. The effect of finite reaction rate on the wind-aided flame propagation speed is found to be similar to its effect on the flame propagation velocity against the flow (see [3], for example).

When the effects of surface radiation losses are included, our numerical analysis shows that the flame spread process is extinguished at high Damköhler numbers; that is, at low velocities flame spread is not possible. The reduction in flame spread velocity due to surface radiation is shown in Fig. 8 in the Burke-Schumann limit $\bar{D}$ $\rightarrow \infty$. Our analysis indicates that flame spread does not occur for values of $\bar{S}_{R}$ larger than, roughly, 0.6. This behavior has also been described for flame spread against the flow over thin fuel slabs in microgravity (see [14] and [17]).

The authors express their appreciation to the European Space Agency and to Comision Interministerial de Ciencia y Tecnologia for the support given to this research under Contracts ESTEC/ 9391NL/JSC and CICYT ESP96-1323-E respectively. We also acknowledge the support of this research by the Human Capital and Mobility Program of the EC, under Contract CHRX-CT940623. 


\section{REFERENCES}

1. Williams, F. A., Sixteenth Symposium (Intemational) on Combustion, The Combustion Institute, Pittsburgh, 1976, p. 1281.

2. Williams, F. A., Combustion Theory, Benjamin Cuminings, 1985, p. 509.

3. Fernandez-Pello, A. C., and Hirano, T., Combust. Sci. Technol. 32:1 (1983).

4. Wichman, I. S., Prog. Energy Combust. Sci. 18:553 (1992).

5. Di Blasi, C., Prog. Energy Combust. Sci. $19: 71$ (1993).

6. Sirigano, W. A., and Schiller, D. N., in Physical and Chemical Aspects of Combustion (F. L. Dryer and R. F. Sawyer, Eds.), Gordon and Breach, New York, 1997, p. 353.

7. de Ris, J. N., Twelfth Symposium (Intemational) on Combustion, The Combustion Institute, Pittsburgh, 1969, p. 241.

8. Williams, F. A., Prog. Energy Combust. Sci. 8:317 (1982).

9. Fernandez Pello, A. C., Combust. Flame $36: 63$ (1979).

10. di Blasi, C., Crescitelli, S., Russo, G., and Fernandez-
Pello, A. C., Twelfth Symposium (International) on Combustion, The Combustion Institute, Pittsburgh, 1988, p. 205.

11. Krishnamerthy, L., and Williams, A., Acta Astronautica 1:711 (1974).

12. Frey, A. E., and T'ien, J. S., Combust. Flame 36:263 (1979).

13. T'ien, J. S., Combust. Flame 65:31 (1986).

14. Olson, S. L. (1987). The Effect of Microgravity on Flame Spread Over a Thin Fuel. NASA TM 100195.

15. Keller, H. B., in Numerical Solution of Partial Differential Equations (B. Hubbard, Ed.), Academic Press, New York, 1971, p. 327.

16. Keller, H. B., Ann. Rev. Fluid Mech. 10:417 (1978).

17. Bhattacharjee, S., and Altenkirch, R. A., Combust. Flame 84:160 (1991).

18. Dwyer, H. A., ALAA J. 22:1705 (1984).

19. Liñan, A., Acta Astronáutica 1:1007 (1974). 